\title{
BIRLIOGRAFIA DEL SECTOR PESQUERIA Y ACUICULTURA \\ CONTINENTAL EN LA AMAZONIA PERUANA
}

Calle Barco Carlos $\left(^{*}\right)$

Alcántara Bocanegra Fernando $\left({ }^{* *}\right)$

1. Alcalá Espinoza Beatriz - 1976: Parásitos de peces de la laguna de Quistococha. U N M SM . Tesis Programa A cadémico de Ciencias Biológicas (27 pág.)

2. Alcántara Bocanegra F. - 1985 Reproducción inducida de gamitana, Colossoma macropomun, Cuvier en cl Perú. U niversidad Nacional de Trujillo - Facultad de Ciencias Biológicas. Tesis D octoral (38 pág.)

3. Alcántara Bocanegra Fernando - 1986: Avances en cl cultivo de gamitana, Colossoma macropomun Cuvier, 1818, en cl laboratorio de Iquitos del IM ARPE. Rey. Lat. A cui. Lima - Perú, N o. 27-27-32-Mar.

4. Alcántara Bocanegra Fernando y Guerra Flores Humberto - 1985: A vances en la producción de alevinos de gamitana, Colossoma macropomun y paco, C. brachypomun por reproducción inducida. Instituto del Mar del Perú (IMARPE) Laboratorio de Iquitos. Revista Latinoamericana de Acuicultura. Lima. No 30 (13 pág.)

5. Alcántara Bocanegra Fernando - 1990: Observaciones sobre el comportamiento reproductivo del paiche, Arapaima gigas, en cautiverio. Instituto de Investigaciones de la A mazonía Peruana (4 pág.)

6. Alcántara Bocanegra Fernando - 1987: Producción de alevinos de gamitana Colossoma macropomun (Cuvier) 1818, mediante hipofisación. Informe interno. Instituto de Investigaciones de la A mazonía Peruana,

Ing. Geólogo- INVESTIGADOR: DIRECCION DE INVESTIGACION DE SUELOS Y AGROINDUSTRIA.

INSTITUTO DE INVESTIGACIONES DE LA AMAZONIA PERUANA

** DIRECTOR DE INVESTIGACION EN RECURSOS HIDROBIOLOGICOS. 
7. Alcántara Bocanegra Fernando, Sánchez Riveiro Homero, M eza Olaechea J orge 1989: Reporte de producción de alevinos de gamitana Colossoma macropomun, en Pucallpa (4 pág.)

8. Alcántara Bocanegra Fernando - 1983 Ensayo preliminar de cultivo mixto de gamitana y paco. IM ARPE, Iquitos.

9. Alcántara Bocanegra Fernando - 1982 Cultivo de paiche A rapaima gigas utilizando bufurqui, Cichlassoma bimaculatum, como presa. Instituto de Investigaciones de la A mazonía Peruana (9 pág.).

10. Alcántara Bocanegra Fernando - 1982: Ensayo preliminar de policultivo de gamitana, sábalo cola roja y boquichico. IM ARPE. Iquitos.

11. Alcántara Bocanegra Fernando - 1980: Ensayo preliminar de cultivo de gamitana, Colossoma macropomun, asociado a la cría de cerdos. IM A RPE.

12. Alcántara Bocanegra Fernando y Guerra Flores Humberto - 1980 A spectos de alevinaje de las principales especies nativas utilizadas en piscicultura en la amazonía peruana. IM ARPE. Iquitos. (28 pág.).

13. Alcántara Bocanegra Fernando - 1983 Primeras observaciones sobre reproducción inducida de gamitana: IM ARPE. Iquitos.

14. Alcántara Bocanegra Fernando - 1983 Contribución al estudio de la reproducción inducida de gamitana, Colossoma macropomum. IM A RPE - Iquitos.

15. Alcántara Bocanegra Fernando - 1982: Ensayo sobre reproducción inducida de boquichico, Prochilodus nigricans Agassiz, 1929, en cautiverio, IMARPE. Iquitos (15 pág.).

16. Alcántara Bocanegra Fernando - Reporte de producción de alevinos de "gamitana", Colossoma macropomun, y "paco", C. brachypomun, en Iquitos. Instituto de Investigaciones de la A mazonía Peruana (IIAP)

17. Alcántara Bocanegra Fernando - : Hibridación de "paco". Brachypomun x "gamitana" Colossoma macropomun, en Iquitos. Instituto de Investigaciones de la A mazonía Peruana (IIAP) 
18. Felipe - 1960: La potencialidad pesquera de nuestra selva y su explotación, PescaCaza №. 10, M inisterio de A gricultura - Lima.

19. A raujo Riveiro, Rafael A ugusto - 1985: Estudio de procesamiento de boquichico, (Prochilodus nigricans), ahumado en caliente. Tesis de grado U NA. (103 pág.)

20. A scón Dionisio Gilberto - 1986 Reproducción artificial de gamitana, Colossoma macropomun y paco, Piaractus brachypomun mediante inducción con hipófisis de tilapia rendalli - IIA P - DIREPE XIII - M oyobamba.

21. A scón Dionisio Gilberto - 1987: Cultivo masivo de rotíferos y cladóceros para la crianza de larvas de peces del género Colossoma en estanques de cemento. IIAP DIREPE XIII - M oyobamba (10 pág.)

22. Ascón Dionisio Gilberto - 1987: Producción de gamitana, Colossoma macropomun, y paco, Piaractus brachypomun, mediante el emipleo de dos técnicas diferentes de reproducción inducida. IIAP - DIREPE XIII - M oyobamba 16 pág.)

23. Ascón Dionisio Gilberto - 1987: Cultivo masivo de rotíferos del género Brachionus como alternativa de supervivencia de larvas de peces del género

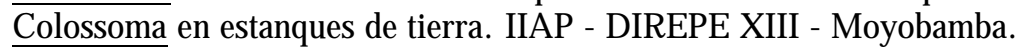

24. A scón Dionisio Gilberto, Campo verde Vigo L uis, L oayza A guilar R. y Saldaña Rojas G. - 1986: Reproducción artificial de gamitana, Colossoma macropomun (Cuvier) 1818, con extracto hipofisiario de "carpa" en el Departamento de San M artín. IIAP - DIREPE XIII - Tarapoto (6 pág.)

25. A zabache Coronado Luis y M aco García José - 1983: Limnología en estanques seminaturales de experimentación piscícola. IMARPE. Iquitos.

26. Azabache Coronado Luis - 1983: Fitoplancton del sistema hidrográfico de la amazonía peruana. IM ARPE. Iquitos.

27. A zabache Coronado L uis, Alcántara Bocanegra Fernando, Maco García José y Guerra Flores Humberto - 1983: Ensayo de fertilización en estanques seminaturales, IM A RPE. Iquitos.

28. A zabache C.L., M aco G.J. y Guerra F.H. 1981: La limnología en la amazonía peruana: química del agua, producción primaria, producción secundaria y otros. IM ARPE - Iquitos. Informe (39 pág.) 
29. A zabache C.L., Nájar A.P. y M aco G.J. 1981: Tipificación de los cuerpos de agua de la amazonía peruana IM A RPE - Iquitos. Informe (32 pág.)

30. Baylcy Peter B. - 1981 Evaluación de la situación actual del proyecto FAO/PER/76/022 en Iquitos y recomendaciones para el mejoramiento de los procedimientos de limnología y biología pesquera. IM ARPE. CERPER Iquitos.

31. Bayley Peter B. - 1981: Características de inundación de los ríos y áreas de captación en la amazonía peruana; su importancia en el sector pesquero:

U na interpretación basada en imágenes de Land Sat e informes de ONERN. IMARPE. Iquitos.

32. Benitez Requena Eduardo - 1977: Contribución al estudio bioecológico del fasaco (pisces erythricidae) en la zona de Pucallpa. UN M SM - (54 pág.)

33. Benitez A raujo F. - 1972: Factor de condición en tilapia melanopleura Dum del lago Sauce durante los meses de febrero - julio de 1972. Tesis de grado UNT. (25 pág.)

34. Berenz Vásquez Mercedes - 1972: Peces de consumo humano de la familia Cichlidae en Iquitos. Tesis de Biólogo-U N A P. (30 pág.)

35. Berger Cebrelli Cristian - 1972: Estudio del factor de condición del paiche, A rapaima gigas (Cuvier), en las zonas reservadas del río Pacaya, entre los años 1959 y 1969. Tesis de grado U N M SM . (60 pág.)

36. Beuzeville Zumaeta J ames - 1973: Los consumidores de pescado en el distrito de Iquitos y su proyección en el mercado. Tesis de Biólogo UNAP.

37. Bohlke James, Weryemann Stanley y Naercio A. M enezes: Estado actual de la sistemática de los peces de agua dulce de la amazonía del sur 1978. Acta A mazónica.

38. Calacho Salas Segundo Esteban - 1975: Efecto de la densidad de población en la crianza de la tilapia (Tilapia rendalli houlanger), en la zona de Tingo M aría. Tesis de grado UNAS (Tingo M aría).

39. Cambero Alva Pedro Ronnier - 1984: Estudio de la producción primaria y biomasa fitoplanctónica de estanques seminaturales del IM ARPE - Iquitos. Tesis de grado UNFV. (37 pág.) 
40. Campos Baca Luis - 1982-1983 Evaluación del potencial pesquero: Huallaga Central - Bajo M ayo.

41. Campos Baca Luis y Castañeda Ruiz Miguel - 1978: Reporte sobre producción piscícola tropical en el Perú.

42. Campos Baca Luis y Padilla Palmira - 1985: Efectos del "kudzú" (Pueraria phaseoloides) y del "Cetico" (Cecropia sp) como fuentes proteicas en la alimentación de "gamitana". Instituto de Investigaciones de la A mazonía Peruana -CIJH. (27 pág.)

43. Campos Baca Luis y Padilla Palmira - 1986: Resultados de un ensayo sobre cultivos de machos y hembras de tilapia nilótica, Sarotherodon nilótica (Linnaeus) en estanques artificiales - IIAP - CIJ H.

44. Campos Baca Luis - 1986: Bioecología de los principales peces de consumo en Jenaro Herrera, Informe Técnico A nual- IIAP.

45. Campos Baca Luis, Padilla Palmira y Mori Luis - 1985: M acrófitas acuáticas y sus relaciones ecológicas con los peces en la amazonía peruana. Jenaro Herrera. Congreso Nacional de Botánica - Iquitos.

46. Campoverde Vigo Luis y Saldaña Rojas Guillermo - 1985: Estudio técnico preliminar para el aprovechamiento pesquero de la laguna “El Naranjal'. Y urimaguas. (41 pág.).

47. Campoverde Vigo Luis y Saldaña Rojas Guillermo - 1985: Primer ensayo sobre crianza de tilapia híbrida Sarotherodon hornorum machos Sarotherodon niloticus hembras) asociado a un cultivo de arroz bajo riego en el Dpto. de San M artín Convenio Univ. Nac. San Martín - DIREPE XIII - Tarapoto (23 pág.)

48. Cánepa La Serna Jorge Roberto - 1981: A vances de la piscicultura tropical en la zona de Pucallpa. Tesis de grado U NFV . (60 pág.)

49. Cánepa J.R. - 1982: Estudio bioecológico del "sábalo cola roja" Brycon erythropterum en el sistema de lagunas Supay y al edaños de J enaro Herrera -Requena. Tesis de Ingeniero. Universidad N acional Federico Villareal. Lima (30 pág. ) 
50. Castañeda E. Héctor - 1977: Contenido estomacal de alevinos de paiche, A rapaima gigas Cuvier, en condiciones de cautiverio. Tesis de grado UNT. (26 pág.)

51. Castañeda Ruiz M iguel y Campos Baca Luis - 1982: A mpliación y creación de estaciones pesqueras en el oriente. M inisterio de Pesquería -M oyobamba.

52. Castillo - Castro Carranza A urea - 1971 Ensayo de piscicultura intensiva con las especies amazónicas Brycon melanopterum Copé 1971 y Brycon erythropterurn Copé 1971. Tesis de grado UNT. (65 pág.)

53. Castellanos Cabrera Roberto - 1977: Determinación de la edad por lectura y medición de escamas en tilapia rendalli boulanger en el lago Sauce - San M artín, Tesis de grado UNT. (37 pág.)

54. Cisneros 1-1. Watson - 1964: Comercio y tendencia del mercado en los productos de la región de la selva peruana. Programa de investigación para el desarrollo U niversidad Nacional A graria.

55. Contreras Guadalupe y Villanueva César - 1983: Fertilización de estanques. Universidad Nacional M ayor de San M arcos - Facultad de M edicina Veterinaria (8 pág.)

56. Contreras Salazar Guadalupe Victoria - 1981: Fertilización de estanques en la amazonía. V. Congreso Nacional de M icrobología - A requipa.

57. Copaira M. y M ontalvo César. 1972. Dimorfismo sexual en el paiche (A rapaima gigas Cuvier, 1929), Rey. mv. Pesq. (IVITA) - 203-207. Univ. Nac. M ay. Sn. M arcos.

58. Cope M.D. - 1870: Contribution to the Ictiology of the Marañón River, Am. Phil. Soc. Vol. 1

59. CORDEU Estudio de factibilidad técnico-económica "D esarrollo de Piscigranjas" - 1984. Corporación de Desarrollo de U cayali - Dirección Zonal de A gricultura y Pesquería ( $N$ o de pág. 25)

60. CORDEU - IVITA: Desarrollo de Piscigranjas - 1984: Estudio definitivo (178 pág.) 
61. Cortez Solis J uan Pedro, 1989: Estudio preliminar de ahumado de pescado con especies amazónicas. Instituto de Investigacioncs de la A mazonia Peruana (18 pag.)

62. Cortez Solis Juan Pedro, 1987 Técnicas artesanales de conservación de los recursos pesqueros en la amazonía peruana. Instituto de Investigaciones de la A mazonía Peruana (17 pág.)

63. Cortez Solis Juan Pedro, 1988: Ensayo dc enlatados de pescado con especies amazónicas. Instituto de Invcstigaciones de la A mazonia Peruana (39 pág.)

64. Cortez Solis Juan Pedro, 1989 Instalación de una planta enlatadora de pescado en Iquitos. Instituto de Investigaciones de la A mazonía Peruana (50 pág.)

65, Cortez Solis Juan Pedro - 1986 Artes y métodos dc pesca en la amazonia peruana. Instituto de investigaciones de la A mazonía Peruana (IIAP) (38 pág.)

66. Cortez Solis Juan Pedro - 1988 Características bromatológicas de doce especies hidrobiológicas de la anuizonía. Instituto de Investigaciones de la Amazonía Peruana (IIA P) (11 Pág.)

67. Corrca Ponce Ovidio - 1980 Explotación pesquera integral a nivel comercial en selva alta. Huallaga Central - Bajo M ayo. Subproyccto: Estudio de mercado de productos hidrobiológicos (estudio de prefactibilidad).

68. Corrca Ponce Ovidio, Castro Tomás, Saldaña Rojas Guillermo, Liendo M artínez Carlos, Santistebán Gerardo, Zapata Teobaldo y Sotero Santos 1981: Explotación pesquera integral a nivel comercial en la selva alta: Huallaga Central - Bajo M ayo (Prefactibilidad). (50 pág.) D epartamento de San M artín.

69. Correa Ponce Ovidio - 1981: Explotación pesquera intcgral a nivel comercial en selva alta "Huallaga Central" - Bajo M ayo. Departamento dc San M artín, 1981 (38 pág.) Vol. 1.

70. Correa Ponce Ovidio, Castro Segundo, Saldaña Guillermo y Liendo Carlos 1982 Explotación pesquera integral a nivel comercial en selva alta Tocache-HuallagaM ayo "Estudio de Productividad". Convenio Dirección Regional de Pesquería Tarapoto. (53 pág.) Vol. V. 
71. Corren Ponce Ovidio, Castro Segundo - 1982: Explotación pesquera integralT ocache-Huallaga-M ayo. Subproyecto: Encuesta piloto sobre consumo y demanda de productos hidrobiológicos microregionales del Huallaga Central-Bajo M ayo y Alto Mayo. 1983 (31 pág.)

72. Correa Ponce Ovidio - 1981: Explotación pesquera integral a nivel comercial en la selva alta -Huallaga Central-Bajo Mayo- Vol VI. Subproyecto: proyección de población por provincias y distritos. Encuesta piloto sobre consumo y demanda de productos hidrobiológicos. Microregión Huallaga Central-Bajo Mayo (36 pág.).

73. Correa P.O. - 1976: Los recursos hidrobiológicos y las pesquerías en aguas continentales (11 pág, 8 cuadros y 4 gráficos) (Primera parte de los recursos naturales renovables de las aguas continentales - $\left.\right|^{\text {er }}$ curso para la formación de oficiales superiores de la policía forestal del Perú). Ministerio de Agricultura, Dirección General de Extracción.

74. Cruz C, Ortega H., Guevara J. y Gutiérrez W. - 1977: Notas bioecológicas de algunos peces de la zona de Pucallpa. UN M SM -IV 1TA.

75. Culquichicón M alpica Zoila, Fukushima Nagaoka M anuel, M alca Carlos, A valos Segundo y Y epes Carlos - 1981: A mpliación y creación de estaciones pesqueras Y urimaguas.

76. Culquichicón Malpica Zoila - 1972: Estudio histológico de ovarios de sábalo (Brycon melanopterum Copé 1872 y B. crythroptcrum Copé 1872 de 4 a 13 meses de edad. Tesis de grado UNT (30 pág.)

77. Chapman D.W . - 1979: Informe del consultor del proyecto FA O-PER/76/- 022 sobre capturas. IM ARPE. Iquitos.

78. Delboy y Dorado Emilio - 1950: Curiosas especies ictiológicas de nuestra amazonía. Bol. Soc.Geog. Tomo LX VII. Lima (18-21 pág.)

79. Diaz M.B., Azabache L.C. y Nájar A.P. - 1980: Avances de los estudios limnológicos en la amazonía peruana con prospección a la tipificación de los cuerpos de agua. IM AR PE - Iquitos, Informe (110 pág.)

80. Dirección de la Pequeña Empresa: Aren de Promoción y Desarrollo: Departamento de Proyectos. Perfil de inversión "Piscigranja', Banco Industrial Tarapoto. 1981 (4 pág.). 
82. DIREPE XIII: Piscigranja comunal de Jepelacio-M oyobambal982. Dirección Regional de Pesquería M oyobambaDIREpE XIII.

83. DIREPE XIII: Piscigranja flotante piloto Alto M ayo 1983. Dirección regional de Pesquería - M oyobamba.

84. DIREPE XIII: Piscigranja piloto rural - Morona - 1982: Dirección Regional de Pesquería XIII - M oyobamba.

85. DIREPE XI - Proyecto 20: Estudio de factibilidad técnico-económico para la creación de la estación de pesquería de A huashiyacu - 1979.

86. Documet M afaldo Teresa - 1977: Estudio preliminar del fitoplancton superficial del lago Quistococha. Tesis de Biólogo UNAP. (75 pág.)

87. Eckman Reiner: The fisheries-situation in the Peruvian amazon region.

88. Eckman Reincr - 1980: Induced reproduction in Prochilodus nigricans from the upper amazon. A qualture V ol. 20 No 9, 381-383 pág.

89. Eigenmann C.H. - 1982: Fishes of Western South American. University of kentucky. Lexington. Vol. 3 (07 pág.)

90. Factor Astocondor Pablo Roque - 1983: Explotación de truchas mediante el empico de jaulas flotantes en la laguna Jillaulla Pozo. Tesis de grado UNFV. (141 pág.)

91. FAO - IMARPE: La pesquería en la amazonía peruana presente y futuro IMARPE, 1982 - Iquitos.

92. Flores Ponce Roberto Pablo - 1978: Estudio de factibilidad técnica en piscicultura comercial para implementarse en la SAIS Tupac A maru Ltda. No 1, con sede en Pucallpa. Tesis de grado UNA. (114 pág.)

93. Fowier Henry W. - 1945: Los peces del Perú - Catálogo sistemático de los peces que habitan en aguas peruanas - U niversidad Nacional Mayor de San Marcos Lima. M useo de Historia N atural Javier Prado.

94. Fukushima Nagaoka M anuel - 1976: Estudio para la determinación del potencial piscícola en el Departamento de San M artín. (62 pág.) Convento MIPE-U NT. 
95. Fukushinia Nagaoka M anuel 1976: Estudio para la determinación del potencial piscícola en el Departamento de San Martín - Estudio limnológico de las quebradas A huashiyacu e Indañe, 1976 (90 pág. ) M inisterio de Pesquería (R egión IV - Oriente) - Universidad Nacional de Trujillo.

96. Fukushima Nagaoka M anuel, Castillo Gilmer y Saldaña Rojas Guillermo - 1976: Estudios para la determinación del potencial piscícola en el Departamento de San Martín - (58 pág.) Convenio M inisterio de Pesquería- Región IV de Oriente Universidad Nacional de Trujillo.

97. Fukushima Nagaoka Manuel y Tresierra Alvarado - 1974: Evaluación de la población del paiche e implementación de un programa de investigación limnológica y pesquera en el lago Sauce (San M artín) (220 pág.) M inisterio de Pesquería - Universidad Nacional de Trujillo.

98. Fukushima Nagaoka Manuel - 1977: Estudio del potencial pesquero del Departamento de San Martín - Estudio técnico de la zona del Alto Mayo (M oyobamba - Rioja - Naranjillo - M arvica]). (66 pág.)

99. Fukushima Nagaoka Manuel - 1979: Explotación pesquera integral a nivel comercial en la selva alta: Bagua, río Cenepa, río Santiago, río A lto M arañón. Dirección Regional de Pesquería - Región XI M oyobamba (203 pág.)

100. Fukushima Nagaoka M anuel, M aco García J osé, M alca A guilar Carlos, Sánchez César y García Rengifo Héctor - 1980: Desarrollo de Piscigranjas. Organismo Regional de Desarrollo de Loreto (Dirección Regional de Pesquería) U niversidad Nacional de Trujillo (101 pág.)

101. Fukushima Nagaoka M anuel, Culquichicón M al pica Zoila y Becerra Díaz Jaime 1980: Desarrollo de zonas fronterizas (Proyecto A 15-16) Estudio limnológico pesquero del lago Pomacocha. Convenio Ministerio de Pesquería (Región XI M oyobamba) - U niversidad Nacional de Trujillo (129 pág.)

102. Fukushima Nagaoka Manuel, Malca Carlos, Avalos Segundo y Culquichicón Malpica Zoila - 1980: Ampliación y creación de estaciones pesqueras Y urimaguas. Proyecto 2811 B-D3 ‘Estudio limnológíco, topográfico y 
edafológico de las alternativas Chambira, Moyobamba y Simuy (Y urimaguas, Alto Amazonas, Loreto). Convenio Dirección Regional de Pesquería ORDELORETO - U niversidad Nacional de Trujillo. (159 pág.)

103. Fukushima Nagaoka Manuel y Campoverde Vigo Luis - 1976: Estudio para la determinación del potencial piscícola en el Departamento de San M artín: 'Estudio limnológico de las quebradas A huashiyacu e Indañe". Universidad Nacional de Trujillo - M inisterio de Pesquería (DIREPE - Región Oriente LV - Oriente).

104. García Paredes Rosa Cristina - 1978 Algunas notas bioecológicas sobre la palometa (Pisces characidae) en la zona de Pucallpa. Tesis de grado UNMSM. (51 pág.)

105. Geisler R; HA. K nóppel and H. Sioli. 1973. The ecology of fresh water fishes in Amazonia. Present an future task of research in Applied Sciences and Development. Vol. 2 Tubingen. Institute for Scientific Cooperation pp.144-62.

106. Gonzáles Saavedra Rosa. 1975. Consideraciones preliminares en el estudio del carnarón de río, M acrohrachium amazonicum (Heller), Tesis de grado (UNAP) 58 pág.

107. Guerra Flores Humberto - 1980: Desarrollo sexual del paiche, A rapaima gigas, en las zonas reservadas del estado (ríos Pacaya y Samiria). IM ARPE. Callao. Inf. $\mathrm{N} \cong 67$.

108. Guerra Flores Humberto, Montreuil Frías Víctor y Villanueva Correa M arle 1983: A vances del programa de evaluación de recursos pesqueros en la amazonía peruana. IM ARPE. Iquitos. (29 pág.)

109. Guerra F.H., P. Cambero A. y H. Sánchez - 1984: Determinación de la captura artesanal en el bajo U cayali, período de media creciente. Proyecto: Potencial Pesquero Regional - Informe de crucero 8412, Diciembre de 1984. Informe interno. Instituto del M ar del Perú - Laboratorio Iquitos.

110. Guerra Flores Humberto - 1971: M adurez sexual y longitud al primer desove del paiche - Arapaima gigas (Cuvier) en la Reserva Nacional Pacaya - Samiria (Departamento L oreto). Tesis D octoral UNT. (22 pág.) 
111. Guevara J., Gutiérrez W., Ortega H. y Vera J. - 1979: Densidad de carga en la población de sábalo cola roja (Brycon erithropterum Copé 1982), en Pucallpa Perú. U niversidad N acional M ayor San M arcos (UNM SM ) - Instituto Veterinario de Investigaciones Tropicales de A ltura (IVITA).

112. Guevara J., Villanueva C., Gutiérrez IV., Ortega H. y Samanez Iris - 1979 Construcción, manejo y mantenimiento de estanques en la estación principal del trópico. IVITA - Pucallpa. IJ NM SM -IV LTA. Pucallpa.

113. Guevara 1., Gutiérrez W., Cruz C. y Ortega H. - 1979: Efecto de fertilización mineral y órganica en la producción de brycon erithropterum en Pucallpa. UNM SM -IV ITA. Pucallpa.

114. Guevara J., Ortega H., Gutiérrez W. y Cruz C. - 1977 Ecología y manejo de estanques de piscicultura en la zona de Pucallpa - Loreto. UNMSM IVITA. Pucallpa.

115. Guevara Juan, Gutiérrez Walter, Villanueva César y Contreras Guadalupe 1983: Proyecto piloto para el desarrollo de piscigranjas. Convenio UNMSM CORDEU-IVITA (23 pág.)

116. Guevara Juan y Samanez Iris - 1982: Alimentación piscícola: Universidad Nacional de San M arcos - F acultad de M edicina V eterinaria (2 pág.)

117. Guevara Juan, Gutiérrez Walter, Ortega Hernán, Villanueva César, Zaldivar Javier, Samanez Iris y Contreras Guadalupe: Cultivo de peces tropicales en Pucallpa- Informe técnico anual 1982 - 1983. Universidad Nacional Mayor de San $M$ arcos - F acultad de M edicina V eterinaria. Convenio UN M SM IVITA - IIAP (66 pág.)

118. Guevara Carrasco Juan - 1974: Estudio sobre el ciclo biológico, ecología, etología y crianza experimental de sábalo (Pisces characidae) en la amazonía peruana. UN M SM (82 pág.) Tesis de grado U N M SM (24 pág.)

119. Guevara Carrasco Juan: Estudio bioecológico del sábalo (Brvcon) en la zona de Pucallpa.

120. Guevara Carrasco Juan y Zaldívar Rodríguez Javier - 1981: Piscigranja demostrativa de la provincia de Coronel Portillo. Primera Edicion. Universidad Nacional A graria - M inisterio de Pesquería. (13 pág.) 
121. Guevara A., Gutiérrez W., Villanueva C. y Derham P.: Cultivo de peces en la amazonía peruana. 2do. Symposium sobre Desarrollo de la A cuicultura en el Perú. Pág. 14i-171.

122. Gutiérrez W., Guevara i., Samanez 1., Ortega H. y Villanueva C. - 1979 Fertilización de estanques en la piscigranja del IVITA, Pucallpa. UNMSM_IVITA. Pucallpa.

123. Gutiérrez W., Samanez 1., Villanueva C., Guevara J., y Ortega H. - 1977 Limnología y productividad en los embalses del IVITA y su área de influencia. UNM SM - IVITA, 1977.

124. Gutiérrez Walter, Villanueva César, Ortega Hernán y Aquino Rolando -1980: Evaluación del potencial de la laguna Imiriam, como modelo para el manejo de otras zonas pesqueras. Corporación Departamental del Desarrollo de U cayali. (15 pág.)

125. Gutiérrez Walter y Samanez Iris - 1980: Alimentación Popular: Universidad $\mathrm{N}$ acional $\mathrm{M}$ ayor de San M arcos - Facultad de M edicina V eterinaria (3 pág.)

126. Gutiérrez Walter y Contreras Guadalupe - 1983: Limnología y productividad en el IVITA y su área de influencia. Universidad Nacional Mayor de San M arcos Facultad de M edicina V eterinaria.

127. Gutiérrez Walter y Guevara Juan - 1981: Ensayo sobre tasas de carga o siembra de estanques en la zona de Pucallpa. U niversidad N acional M ayor de San M arcos - Facultad de M edicina V eterinaria (2 pág.)

128. Gutiérrez Alva Félix y Ortega Torres Teófilo - 1972: Estudio ictiofaunístico del JVITA (Pucallpa) y sus al rededores, como inicio de una piscicultura con especies regionales. UN M SM . (125 pág.)

129. Gutiérrez Aiva Félix Walter - 1979: Efectos de la densidad de carga sobre la producción de sábalo cola roja (Brycon erythropterum). Rey. Lat. A cui. - №.1.

130. Ismiño Orbe Rosa - 1984: Estudios limnológicos en la cocha Pastor de Padre Isla. Iquitos. Tesis de grado. Universidad Ricardo Palma. Lima.

131. Ismiño Orbe Rosa, M ontreuil Frías Víctor, Maco García José, Tello M artín Salvador - 1990: Plancton de los ríos U cayali y Marañón. Instituto de Investigaciones de la A mazonía Peruana (51 pág.) 
132. Hanck G. (Ed.) - 1982 Pesquería en la amazonía peruana, presente y futuro. FA 0-IM A RPE/PER 176/022. Roma (86 pág.)

133. Kullander 5. - 1981: Key to the cichlid species of the Peruvian A mazonas dreinage sistem. Swedish M useum of $\mathrm{N}$ atural History (6 pág.)

134. Limo Silva Esther - 1978 Aspectos bioecológicos de las lisas (Pisces anostomidae) de la zona de Pucallpa. Tesis de grado UN M SM . (70 pág.)

135. L oayza A guilar Rómulo: Efecto de la densidad de carga y proporción por especie en el policultivo de tilapia híbrida Oreochromis nilolicus x 0 . hornorum e híbrido de gamitana x paco, Colossoma macropomun, con cl uso de gallinaza. 1987 IIAP-DIREPE XIII - M oyobamba.

136. L oayza A guilar Rómulo 1987 Efecto del uso de tres dosis de "cerdaza" en la crianza de "tilapia" híbrida Orcochromis nilotica x 0 hornorum. IIAP - DIREPE XIII, Tarapoto (12 pág.)

137. Loayza Rómulo y Campoverde L. - 1987: Efecto de diferentes dosis de "gallinaza" y "cerdaza" en la fertilización inicial y diaria de estanques piscícolas. Convenio IIAP - DIREPE XIII - Tarapoto.

138. L oayza A guilar Rómulo - 1987: Crecimiento de "tilapia" híbrida Oreochromis niloticus x 0 hornorun y de "Tilapia nilotica' revertida Oreochromis niloticus con el empleo de estiércol de gallinas (19 pág.)

139. Loayza Aguilar Rómulo y Campoverde Vigo Luis, Sotero Montero A., Fasanando del Aguila Julio - 1986: Determinación del dimorfismo sexual y proporción por sexos en las especies "tilapia nilotica" Oreochromis niloticus y "Tilapia hornorum" Oreochromis hornorurn. IIAP-DIREPE XIII - U niv. Sn. Martín - Tarapoto (13 pág.)

140. L ozano A ngulo Linder - 1967: Causas que hacen disminuir la pesca en la selva (río U cayali). Tesis de grado U N M SM - F acultad M edicina V eterinaria (53 pág.)

141. Llerena Horna César - 1981 Peces de consumo humano de los géneros Brycon, Colossoma, Piaractus y Triportheus. Tesis de grado UNT.

142. M artínez M . - 1984 El cultivo de las especies del género Colossoma en A mérica L atina. FA 0. Santiago de Chile (46 pág. ). 
143. M atos Luján José y Zavaleta A guilar Sabino - 1980 Estudio de factibilidad técnico - económico de la piscigranja piloto OASIS. Dirección Regional XI M oyobamba. M inisterio de Pesquería. Dirección Zonal Tarapoto - Unidad de Planificación (DIREPE-XJ). Tarapoto (60 pág.)

144, M atos J, - 1981: Fomento de la acuicultura en San Martín. 2do. Symposium sobre desarrollo de la acuicultura en el Perú. Lima (pág. 173-185)

145, M enchola A cuña A Ivarado - 1977 Evaluación del nivel técnico de procesamiento de las principales especies de la zona reservada de M azán. IM ARPE, Iquitos.

146. M estanza Díaz Tito Orlando 1942: Bases hidrobiológicas para la extracción de la trucha de la cuenca del río M arañón. Tesis de grado U NFV . (77 pág.)

147. M ontreuil Frías Víctor - 1984: La pesquería en la amazonía peruana, avances de la investigación efectuada por el IM ARPE - Iquitos.

148. Montreuil F. Víctor, A. Castañeda, M. Rodríguez, R. Pozo. C. de la Cruz 1980: Diagnóstico de la pesquería en la región amazónica (L oreto-U cayali) IIAPIquitos (128 pág.)

149. M ontreuil Frías Víctor 1-1. y Tollo M artín Salvador - 1987: Determinación de edad en boquichico (Prochilodus nigricans Agassiz, Teleostei: Characoidei) mediante lectura de esclerites en las escamas. Instituto de Investigaciones de la A mazonia Peruana. (IIAP) (19 pág.)

150. M ontreuil F, Víctor - 1986: Extracción, conservación y mercadeo de productos pesqueros en la amazonía peruana - 1986. Instituto de Investigaciones de la A mazonía Peruana (IIAP).

151. M ontreuil F. Víctor y Tollo M artín Salvador - 1986: Inventario y evaluación de los recursos hidrobiológicos en la cuenca de los ríos U cayali y M arañón Informe anual 1986 - Instituto de Investigaciones de la A mazonía Peruana (IIA P).

152. M ontreuil Frías Víctor, M aco García J osé, Tollo M artín Salvador, Ismiño Orbe Rosa, Sánchez Riveyro Homero: Cuadro ambiental de la cocha Carocurahuante y las posibilidades de explotación de camarón del río Macrobrachium amazonicum). 
153. M ontreuil Frías Víctor Hugo, M aco García José, Tello M artín Salvador, Ismiño Orbe Rosa, Sánchez Homero 1987: Estudio de los recursos hidrobiológicos en la amazonía peruana. Instituto de Investigaciones de la A mazonía Peruana.

154. M ontreuil Frías Víctor - 1989: La administración y desarrollo de la pesquería como una alternativa económica para la región del A mazonas IIAP.

155. M ontreuil Frías Víctor Hugo, Tollo M artín Salvador, M aco García José, Ismiño Orbe Rosa - 1989: Rendimiento máximo sostenible de la pesquería comercial en el Departamento de Loreto (25 pág.)

156. M ontreuil Frías Víctor H., Campos Baca Luis - 1988: Diagnóstico de la pesquería en M adre de Dios. Instituto de Investigaciones de la A mazonía Peruana (IIAP)

157. M ontreuil Frías Víctor H., Campos Baca Luis - 1988: Estudios hidrobiológicos en M adre de Dios. Instituto de Investigaciones de la A mazonía Peruana (IIAP)

158. M ontreuil Frías Víctor H. - 1989: Pesquería de la arahuana (Osteoglossum bicirrhosum). Instituto de Investigaciones de la A mazonía Peruana (IIAP)

159. Montreuil Frías Víctor H. - 1989: Estado actual de la pesquería de peces ornamentales de la amazonía peruana. Instituto de Investigaciones de la A mazonía Peruana (IIAP)

160. M ontreuil Frías Víctor H. - 1989: M anejo de recursos pesqueros en la Reserva Nacional Pacaya-Samiria. Instituto de Investigaciones de la A mazonía Peruana (IIAP)

161. M orín L udeña A nibal Waldemar - 1975: Estudio de adopción y alimentación de la tilapia (Tilapia rendalli) en Tingo María. Tesis de Grado UNAS (Tingo M aría).

162. M oro Sommo Jaime - 1964: M étodo biológico para la determinación de residuos de rotenona en peces capturados con cubo o barbasco - Tesis Bachiller U N M SM - Facultad de M edicina V eterinaria.

163. M oscoso Julio - 1975: M ejoramiento genético por selección nasal. Universidad Nacional A grícola La Molina (UNA). Lima.

164, M oscoso Cavallini J. C. - 1983: Ensayo de alimentación artificial bajo diferentes niveles proteicos en la crianza intensiva de la carpa (Cyprinus carpio L.) 
Tesis de grado UNA. (146 pág.)

165. Moscoso A. 1981: Desarrollo piscícola de la selva central (La Merced) 2 do. Symposiurn sobre Desarrollo de la A cuicultura en el Perú. Pág. 187-194.

166. M uñoz Correa Hugo - 1969: A spectos generales de la ictiofauna selvática en el Perú, Tesis de grado UNFV (60 pág.)

167. Nakashime S. 1941-43: Algunos peces del oriente peruano - M useo de Historia $\mathrm{N}$ atural J avier Prado, Boletín № 16.

168. Nakashime S.: N ota sobre la piscicultura del paiche. Boletín $\mathrm{N} N 24-25$. M useo de Historia Natural Javier Prado,

169. Nava Cueto II. L. - 1983: Alimentación artificial bajo diferentes niveles de proteína de la crianza intensiva de tilapia rendalli B. (Melanopleura). Tesis de grado UNA. (129 pág.)

170. Nicho Carpio Eddy y Villareal Zavaleta Wilner - 1984: Crianza intensiva carpa común (Cuprinus carpio) con fines comerciales en el lago Pomacocha A mazonas. Dirección Zonal de Pesquería - Chachapoyas. M inisterio de Pesquería Dirección Regional de Pesquería II - Cajamarca. Chachapoyas.

171. N oé Norma y Silos Gonzáles del A guila - 1983: Estudio microbiológico del agua y de los peces de los estanques de piscicultura del IV ITA. U niversidad Nacional M ayor de San M arcos - Facultad de M edicina V eterinaria (4 pág.)

172. Obando Llejaruria Carlos - 1974: Contenido estomacal de tilapia rendalli Dum del lago Sauce (San M artín) durante el segundo semestre de 1974 Tesis de grado UNT. (27 pág.)

173. Oficina Sectorial de Planificación - M inisterio de Pesquería: La pesquería en la región selvática. M inisterio de Pesquería - 1971 (135 pág.)

174. Ortega Hernán, V illanueva César, Samanez Iris y Contreras Guadalupe Elementos básicos de piscicultura tropical. IVITA - Pucallpa.

175. Ortega Hernán - 1981: Estudio bioecológico de las principales especies ícticas comerciales de la zona de Pucallpa. U niversidad Nacional M ayor de San M arcos - Facultad de M edicina V eterinaria. 
176. Ortega Hernán y Samanez Iris - 1982 Estudio sistemático de los peces de la amazonía peruana y la colección ictiológica de la UNMS. U niversidad Nacional M ayor de San M arcos - F acultad de M edicina V eterinaria (9 pág.)

177. Ortega Hernán y Contreras Guadalupe - 1982: Estudio bioecológico de las principales especies ícticas de la amazonía peruana. U niversidad Nacional Mayor de San M arcos - Facultad de M edicina V eterinaria (7 pág.)

178. Ortega de Palti M artha, Caballcro de Sánchez Rosa y Rado E. 1975 A islamiento y purificación de ácidos ribonucleicos y desoxiribonucleicos de gonadas dc peces. Universidad Nacional Mayor de San Marcos, 1975, X Congreso Panamericano Farmacia y Bioquímica - Punta del Este - U ruguay.

179. Ortega Hernán y Samancz Iris - 1983: Estudio bioecológico de las principales especies de peces de consumo en la amazonía peruana. U niversidad Nacional M ayor de San M arcos - Facultad de M edicina V eterinaria (9 pág.)

180. Ortega 11. y Van R. - 1985 : A nnotated Checklist of the Fresh Water Fishes of Perú. Smithsonian Contributions lo Zoology.

181. Ortega T.H., Cruz P.C., Gutiérrez A. W. y Guevara C.L.: Ictiofauna de la zona de Pucallpa. Loreto. Convenio M inisterio de Pesquería - IVITA. Pub, № 30 (71 pág.)

182. Padilla Pérez Palmira - 1986: Ciclidos del sistema de lagunas Supay y bioecología del "Bujurqui vaso" Chactobranchus flavescens Heckel - 1840, en Jenaro Herrera - Loreto. Tesis de grado Biólogo-UNAP.

183. Paredes Pérez Rodolfo - 1984: Problemática de la pesquería continental en el Perú - Simposio Nacional de Pesquería Continental. Colegio de Ingenieros del Perú - Capítulo de Ingenieros Pesqueros:

184. Pezo Díaz Roberto y Sicchar Valdez Luis - 1979: Reproducción inducida por hipofisación en "Boquichico" Prochilodtis nigricans Agassiz 1929. Tesis de Biólogo, UNAP. (33 pág.)

185. Pezo D. Roberto, M ontreuil Frías Víctor H., M aco García José, Tello M artín Salvador, Cánepa La Serna Jorge, Cortez Solis Juan 1985 Programa de investigaciones y desarrollo pesquero. Instituto de Investigaciones de la A mazonía Peruana (IIA P) (157 pág.) 
186. Piazza L, Alvaro y Vildoso A belardo - 1964 Pesquerías: Problemas y soluciones para su fomento en la selva. Fórum de selva - instituto de Selva - Universidad Nacional A graria. Vol. 1.

187. Pinedo del Aguila V.: Evaluación económica de los recursos ícticos de la amazonía peruana. Facultad de Ciencias E conómicas - U N M SM .

188, Pinto Pérez César Antonio - 1982: Evaluación y crecimiento de especies tropicales introducidas bajo el sistema de policultivo. Tesis de grado UNA. (156 pág.)

189. Pizarro Baldión Juan Alberto. -1976: Actividades de la pesca en la zona de reserva del río Pacaya - Departamento de Loreto. Tesis de grado UNFV. (37 pág.)

190. Proyecto 1816-22: Explotación Pesquera Integral Tocache-Huallaga y Mayo Documento No 5. Informe del reconocimiento preliminar de las actividades pesqueras en la cuenca del río Tocache y Alto Huallaga (Huallabamba Tocache). Informe de viaje. Dirección Regional de Pesquería XI, M oyobamba-Tarapoto-San M artín.

191. Proyecto 1816 - 22: Explotación Pesquera integral Tocache Huallaga y M ayo: Informe del reconocimiento preliminar de las actividades pesqueras en la cuenca del Bajo Huallaga (Shapaja-Papaplaya). Documento No. 6 - Informe de viaje. Dirección Regional dc Pesquería XI - M oyobamba. Tarapoto - San M artín.

192. Proyecto 1816 - 22: Explotación Pesquera Integral Tocache - Huallaga y M ayo Documento de trabajo $\mathrm{N}$ ‥8. Informe de viaje: Informe de las actividades del centro de operaciones pesqueras 'Paso del Vaquero', Evaluación de la limnología y biología de la pesca. Dirección Regional de Pesquería XI, Moyobamba Tarapoto - San M artín.

193. Proyecto 1816 - 22: Explotación Pesquera Integral Tocache - Huallaga y M ayo D ocumento de trabajo No. 10 - Informe de actividades: A ctividades del centro de operaciones pesqueras Rioja (noviembre). Incremento y desarrollo pesquero A huashuyacu- Evaluación de los afluentes del Alto $M$ ayo durante el mes de noviembre de 1979. Dirección Regional de Pesquería XI- Moyobamba, Departamento de San M artín. 
194. Proyecto 1816 - 22: Explotación Pesquera Integral Tocache - H uallaga y Mayo - Documento de trabajo №. 11 Avances de la evaluación del potencial hidrobiológico de la cuenca del Alto M ayo durante el mes de diciembre de 1979. Incremento pesquero de Neshuya. Dirección Regional de Pesquería XIM oyobamba. Tarapoto - 1979.

195. Proyecto 1816 - 22: Explotación Pesquera Integral Tocache-Huallaga-M ayo. Documento de trabajo No. 12: A vances de la evaluación del potencial hidrobiológico de la cuenca hidrográfica del Huallaga Central (Picota Sabaloyacu), durante el período julio-diciembre 1979 - Dirección Regional de Pesquería XI - M oyobamba - Tarapoto.

196. Quiroz Alcalde Alberto - 1984: Artes y técnicas de pesca utilizadas en la amazonía peruana - Simposio Nacional de Pesquería Continental. Colegio de Ingenieros del Perú - Capítulo de Ingenieros Pesqueros.

197. Quiroz A. Alberto y Saravia T. Pablo - 1979: Evaluación de las artes y métodos de pesca empleados en el río M azán. IMARPE. Iquitos.

198. Ráez Oyola Víctor M anuel - 1976: Algunos aspectos sobre el ciclo biológico, ecología y cultivo del Prochilodus nigricans Agassiz - Pucallna, Tesis de grado UNM SM. (661 pág.)

199. Regan C.T. - 1906: A revision of the fishes of the South American Cichlid. Género Cichla, Chaeotebranchus and Chaetobranchopsis with notes of the A merican Cichlids. A nn. M ag. M at. Hist. Vol. 7.230 (239 pág.)

200. Rivera Chávez Pedro L .: La pesca en el U cayali Central (23 pág.)

201. Rodríguez Navarro M anuela - 1972: Peces de la familia characidae en la ciudad de Iquitos. Tesis Título de Biólogo - UN AP - Iquitos (45 pág.)

202. Rodríguez Díaz J osé M aría del Carmen - 1983: A spectos técnico- económicos de la explotación de los recursos naturales en el Departamento de San M artín. Tesis de grado UNFV. (56 pág.)

203. Rojas Sánchez Danilo - 1976: Contenido estomacal de tilapia rendalli Dum del lago Sauce (Departamento de San M artín), durante los meses de julio diciembre de 1976. Tesis de grado (42 pág.) UNT.

204. Rojas Vásquez José y M ori P. Luis - 1976: Especies ícticas más comunes de la provincia de M aynas. Primera Edición UNAP. (22 pág.) 
205. Rojas Vásquez José - 1974: Estudio preliminar de la especie Hypbossohrycon inmesi "Tetra neón o Paiba".

206. Rojas Vásquez José, Siva Tccco Gilberto, Braga Vela Janeth - 1979 Estudio preliminar del Macrobrachium amazonicum "camarón de río" (Heller). UNAP, (71 Pág.)

207. Rojas Vásquez José 1977 Estudio preliminar de las especies Curimata rutiloides 'Ractacara", L eporinus ovolfei Liza" y Trachy coryster galatus 'novia'.

208. Ruíz Frías Angel - 1974: Enfermedades más comunes de peces ornamentales reportados de los acuarios comerciales de la ciudad de Iquitos. Tesis de Biólogo UNAP. (66 pág.)

209, Ruíz Ríos L eoncio, Saenz Ganoza A lvaro, León Carreño J osé, V illanueva Torres César: Reproducción del boquichico Prochilodus nigricans) Agassiz (1829) por medio de hormonas. U niversidad Nacional A graria - Fundación Internacionl para la Ciencia de Suecia (33 pág.)

210. Saldaña Rojas Guillermo - 1980: Plan regional para el desarrollo de la piscicultura tropical en el Departamento de San Martín. Primera Parte: Microregión Huallaga Central - Bajo Mayo. Ministerio de Pesquería - DIREPE XI (412 pág.).

211. Saldaña Rojas Guillermo - 1984: Ensayo sobre reproducción inducida de 'gamitana', Colossoma macropomun (Cuvier 1818), con gonadotropina coriónica humana. Tarapoto (Rey. Hidrobios 1-12 pág.).

212. Saldaña Rojas Guillermo y Castañeda Ruíz Miguel - 1978: A porte de la tecnología de transporte de alevinos de paiche, Arapaima gigas Cuvier. M inisterio de Pesquería, Sauce - Tarapoto (14 pág.)

213. Saldaña Rojas Guillermo, Pichilingue N. Víctor, Sánchez Jorge - 1986 Efectos de la alimentación proteíca en el crecimiento de "tilapia" híbrida Oreochromis hornorum macho $x$ Oreochrornis niloticus hembra en estanques seminaturales, IIAP - DIREPE XIII - Tarapoto (8 pág.)

214. Samanez 1., Gutiérrez W., Guevara J., Villanueva C. y Ortega H. - 1979 Estudios preliminares sobre reproducción artifical de las especies en cultivo. UNM SM -IV ITA, 1979. 
215. Sánchez 1. y Ancieta C. Felipe - 1946: Limnología y piscicultura en la selva peruana - plancton de la cocha Zapote. Ministerio de Agricultura Dirección de Pesquería.

216. Sánchez R. Jorge - 1951: Posibilidades pesqueras en la selva peruana. A nuario de las industrias pesqueras del Perú.

217. Sánchez R. Jorge - 1951: Peces peligrosos en los ríos y lagos de la amazonía peruana - 1951. Pesca y Caza № . 2. M inisterio de A gricultura - Lima.

218. Sánchez R. Jorge - 1961: EI paiche (A rapaima gigas), Aspectos de su historia natural, ecología natural. Ecología y aprovechamiento. Revista Caza y Pesca № 10. Dirección de Pesquería y Caza, M inisterio de A gricultura. Lima (pág. 1763).

219. Sánchez Romero Jorge - 1960: EI paiche (A rapaima gigas) y su ambiente. Tesis de grado UN M SM . (30 pág.)

220. Sánchez Y actayo Saul Salvador - 1980: Evaluación de las posibilidades de desarrollo piscícola de las provincias de Tarapoto y Tahuamanu (Dpto. de M adre de Dios). Tesis de grado UNA. (18 pág.)

221. Sato Alberto, Guevara Juan y Gutiérrez Walter - 1980: Estudios preliminares sobre producción artificial de las especies en cultivo. Convenio UN M SM IVITADPCB (6 pág.)

222. Sato Alberto, Guevara Juan y Gutiérrez Walter - 1980: Método mixto de piscicultura como tipo de cría asociada con otras actividades pecuarias en Pucallpa. Universidad Nacional Mayor de San Marcos - Facultad de Medicina Veterinaria (Convenio UN M SM -IVITA -D PCB (5 pág.)

223. Sato Alberto, Guevara Juan y Gutiérrez Walter - 1980: Construcción, manejo y mantenimiento de estanques de la estación principal de trópico JVITA - Pucallpa. U niversidad Nacional M ayor de San M arcos - Facultad de M edicina $V$ eterinaria (Convenio IV 1TA-CPCB-DIREPO-DGRCT- ORDELORETO (8 pág.)

224. Sato Sato Alberto, Guevara Carrasco Juan y Gutiérrez Alva Walter - 1980: Cultivo de peces tropicales en Pucallpa - Universidad Nacional Mayor de San Marcos - Facultad de Medicina Veterinaria (88 pág.) Convenio UNMSM, IVITA, DPCB (Departamento de Ciencias Biológicas) - M inisterio de Pesquería, DIREPO, DGICT. 
225. Solano J. - 1973: Reproducción inducida del “Boca chico' Prochilodus reticulatus $\checkmark$ alenciennes. Simposio internacional sobre fauna silvestre y pesca fluvial 0 lacustre amazónica. (34 pág.)

226. Soregui V argas Juan - 1981: Algunas consideraciones de los recursos ícticos de la zona reservada del río Pastaza. UNAP - Tesis Programa Académico de Ciencias Biológicas (88 pág.)

227. Tello Jorge Salvador - 1981: Estudio técnico-económico para la instalación de una piscigranja de gamitana Colossoma macropomun), en la localidad de Tarapoto. Universidad Nacional Federico Villareal - Programa Académico de Oceanografía y Pesquería. Tesis Ingeniero Pesquero U NFV. (95 pág.)

228. Torres M aldonado Rosa Margarita - 1974: Contenido estomacal del "dorado" Brachyplatystoma flavicans (Castelnau 1885). T esis de Biólogo U NAP. (28 pág.)

229. Tovar Cerpa Augusto - 1967: Peces del Oriente Peruano - Algunas especies de loricaria, con referencia especial de la "Carachama" Pterygonlichtis multiradiatus (Hancok), Ecología y utilidad-Biota. Vol. No. VI No. 50. Museo de Historia $\mathrm{N}$ atural J avier Prado - Lima.

230. Tovar Serpa Augusto - 1966: Peces del Oriente Peruano. Tesis de grado UNM SM . (88 pág.)

231. Tresierra A guilar Alvaro - 1974: Algunos aspectos de la biología y ecología del "paiche", A rapaima gigas C., del lago Sauce (D pto. San M artín). Tesis Doctoral UNT. (35 pág.)

232. Tresierra A guilar Alvaro - 1976: Factor de condición de tilapia rendalli Dum en el lago Sauce (Departamento de San M artín). Tesis de grado UNT. (29 pág.)

233. Universidad Nacional M ayor de San Marcos - Líneas de Piscicultura Tropical y Nutrición Animal: Alimento suplementario y técnica de alimentación en la producción de peces en Pucallpa, 1980. Universidad Nacional Mayor de San Marcos - Facultad de Medicina Veterinaria (7 pág.) Convenio IVITA-DPCBDIREPE-DGIC, 
234. Universidad Nacional M ayor de San M arcos - F acultad de M edicina V eterinaria Estudios bioecológicos de las principales especies comerciales de la zona de Pucallpa. Convenio UN M SM FMV - 1980 (8 pág.)

235. Universidad Nacional M ayor de San M arcos - Facultad de M edicina Veterinaria Estudio de la ictiofauna del IVITA y su área de influencia y formación de la colección de peces del oriente peruano. Convenio U NM SM - 1980 (7 pág.)

236. UNM SM - Departamento A cadémico de A natomía e Histología - UNM SM 1975 Dimorfismo sexual en el paiche, (A rapaima gigas (Cuvier) 1929. Documento No 55 (M 1PE) 30-34,

237. Universidad Nacional de Trujillo - 1973 Evaluación de la población del paiche e implementacion de un programa de limnología pesquera en el lago Sauce D ocumento - M inisterio dc Pesquería-Lima - año III n: 35-36 (pág. 55- 58).

238. Vallejos Vega Héctor y Menchola A cuña Alvaro - 1984 Composición y valor nutritivo de algunas especies pesqueras continentales - Colegio de Ingenieros del Perú - Capítulo de Ingenieros Pesqueros.

239. Vásquez M.M. - 1973 Peces ornamentales de la amazonía peruana. Universidad N acional de la A mazonía Peruana - Iquitos (166 pág. ).

240. Vela Guerra Luz Esther y Carey Solís Pedro Adolfo - 1984 Dismidias de los estanques No 1, 5, 9, 10 y 11 de la piscigranja Quistococha. Universidad $\mathrm{N}$ acional de la A mazonía Peruana (U NAP) - Iquitos.

241. Venturi Victor - 1975 Cultivo dc camarones de estanques - bioecología, crianza y repoblación. U niversidad N acional A graria La M olina (UNA). 1975.

242. Villacorta Correa M arie - 1981 Lista de peces identificados en el laboratorio del IMARPE - Iquitos. Informe Técnico. Iquitos.

243. Villacorta Correa Marle - 1976 Algunas consideraciones bioecológicas del "Churo" Pomacea maculata Perry. U N A P. Tesis Título Biólogo (68 pág.)

244. Villacorta A. 1986 Estudio experimental de elaboración de enlatados de pescado tipo "grated" a partir de la especie amazónica "Boquichico", Procchilodus nigricans. Ed. rey. Universidad Nacional de la A mazonía Peruana, Iquitos (90 pág.) 
245. Villanueva C., Gutiérrez XV., Samanez 1., Ortega H. y Echevarria H. - 1979 Alimento suplementario y técnica de alimentación empleada en producción de peces en Pucallpa. U N M SM - IVITA, 1979-Pucallpa.

246. Villanueva C., Ortega H., Guevara J. y Samanez 1. - 1980: Estudio bioecológico de las principales especies comerciales en la zona de Pucallpa. U NM SM -IVITA. Pucallpa.

247. Villanueva César y Guevara Juan - 1981: Alimento suplementario y técnica de alimentación empleados en la producción de peces en Pucallpa. Universidad Nacional M ayor de San M arcos - Facultad de M edicina V eterinaria (2 pág.)

248. Villanueva César, Samanez Iris y Contreras Guadalupe - 1983 Estudio preliminar sobre reproducción artificial de las especies amazónicas en cultivo Universidad $\mathrm{N}$ acional M ayor de San M arcos - Facultad M edicina V eterinaria (12 pág.).

249. Villanueva César y Guevara Juan 1982: Ensayo sobre siembra de peces en estanques en la zona de Pucallpa.

250. Villanueva César y Guevara Juan - 1983: Método mixto de piscicultura (Policultivos), como tipos de cría asociada a otras actividades agropecuarias en la zona de Pucallpa - Universidad Nacional Mayor de San Marcos. Facultad de M edicina $V$ eterinaria (4 pág.)

251. Villanueva César, Guevara Juan y Gutiérrez Walter - 1979: La piscicultura tropical en Pucallpa, perspectivas de desarrollo. Colegio de Ingenieros del Perú. Forum Desarrollo del Trópico Húmedo.

252. Villanueva Chávez César A ugusto - 1982: Fertilización de estanques de peces en la amazonía peruana. Symposium Microbiología y Piscicultura. V Congreso Peruano de M icrobiología y Parasitología.

253. Villanueva Chávez César A ugusto - 1979: Los peces de agua dulce del Perú; lista sistemática preliminar de especies. VI Congreso Nacional de Biología - Chiclayo.

254. Villanueva Chávez César A ugusto - 1982: Fertilización de estanques de peces en la amazonia peruana. Symposium Microbiología y Piscicultura, V Congreso Peruano de M icrobiología y Parasitología.

255. Villanueva Chávez César A ugusto - 1979: Efecto dc la densidad de carga en la producción de 'gamitana", Colossoma macropomum, en Pucallpa, VI Congreso Nacional de Biología - Chiclayo. 
256. Villanueva César y Gutiérrez Walter - 1980: Cultivo de peces tropicales en la amazonía. Seminario proyectos de investigaciones ecológicas para el manejo de los recursos naturales renovables del bosque tropical húmedo - Iquitos. ORDELORETO - COTESU.

257. Villanueva César, Gutiérrez Walter y Guevara Juan - 1981 Cultivo de peces en la amazonía peruana. Symposiurn sobre desarrollo de la acuicultura en el Perú. (13 pág.)

258. Villareal Zavaleta Wilmer, Nicho Carpio Eddy, Hidalgo Auberto y Aguilar Maria Elena - 1984: Crianza intensiva de la especie carpa común (Cyprinus carpio) con fines comerciales en el lago Pornacocha-A mazonas. Ministerio de Pesquería (Dirección Regional de Pesquería 11 - Cajamarca. Dirección Zonal de Pesquería - Chachapoyas).

259. Villareal Zavaleta Wilmer - 1979: A provechamiento de los recursos de agua y tierra para el desarrollo de la acuicultura en el Perú. Documenta. Dic. Lima (79 pág.)

260. Wilhelm Mori Elmer - 1975: Aspectos hidrobiológicos de los ríos Itaya, A mazonas y $\mathrm{N}$ anay. Tesis Doctoral (43 pág.)

261. Wismar, R.C. Richey. R.F. Stallard R.F. y J. M. Edmond - 1980: M etabolismo de plancton e ciclo de carbono no río amazonas, seus tributarios e aguas de várzea Perú - Brasil. M ayo-Junio, A cta A mazónica.

262. W osnitza Claudia, Tresierra A Ivaro y Fukushima M anuel - 1976: Estudio para la determinación del potencial piscícola en el Departamento de San Martín "Estudio de la biología y dinámica de la población de tilapia rendalli en el lago Sauce" (80 pág.). Universidad Nacional de Trujillo - Dirección Regional de Pesquería, Región IV.

263. Wosnitza Claudia y Dávila Felix - = 1978: M anual de dinámica de poblaciones de peces. Universidad Nacional de Trujillo - Departamento de Ciencias Biológicas. 